\title{
Kontroversen der axillären Lymphonodektomie (SLN)
}

\author{
H. Eidtmann \\ Universitäts-Frauenklinik Kiel
}

\section{Einleitung}

Nach wie vor ist die axilläre Lymphonodektomie mit Entfernung von mindestens 10 Lymphknoten Standard in der Primärbehandlung des nicht-metastasierten Mammakarzinoms. Dabei ist der Lymphknotenstatus immer noch der wichtigste Prädiktor im Hinblick auf ein Rezidiv oder das Überleben. Geht man davon aus, daß etwa 50-60\% der heutzutage primär diagnostizierten Mammakarzinome nodal-negativ sind, so bedeutet dies bei etwa 46.000 Neuerkrankungen pro Jahr in der Bundesrepublik Deutschland, daß sich etwa 25.000 Patientinnen mit tumorfreien axillären Lymphknoten einer Lymphknotendissektion unterziehen müssen - möglicherweise ohne einen therapeutischen Nutzen für die Patientin. Im Gegenteil, die wesentliche, langfristige Morbidität nach brusterhaltender Therapie mit der Ausbildung von Lymphödemen, Bewegungseinschränkungen des betroffenen Armes sowie Parästhesien und Schmerzen ist bedingt durch die Behandlung der Axilla. Eine weniger radikale Axilladissektion, oder gegebenenfalls in selektierten Fällen der Verzicht auf eine Axilladissektion, könnte also eine langfristige Verbesserung der Lebensqualität der Patientin nach Primärbehandlung eines Mammakarzinoms bedeuten. Notwendig hierzu wäre eine hinreichend sichere Prädiktion eines negativen Lymphknotenstatus. Nach den bisher vorliegenden Daten (s. u.) kann die gezielte Entnahme der ersten das Tumorbett drainierenden Lymphknoten (die sogenannten Sentinel-Lymphknoten, SNL) mit sehr hoher Wahrscheinlichkeit einen negativen axillären Nodalstatus vorhersagen. Noch nicht geklärt ist allerdings, ob die Axilladissektion selbst, also auch bei histologisch negativer Axilladissektion, einen prognostischen Einfluß auf das weitere Überleben der Patientin mit primärem Brustkrebs hat.

\section{Die Standard-Axilladissektion}

Der Standard der Axilladissektion besteht heute in der vollständigen Entfernung des Fett-Lymphknotengewebes aus Level I und II, optional auch aus Level III. Anatomische Leitstruktur ist neben der Vena axillaris vor allem der Musculus pectoralis minor: Alles, was lateral und unterhalb der Vena axillaris liegt, entspricht Level I. Das Gebiet unter der Vene und dem Pectoralis minor entspricht Level II, alles medial vom Pectoralis-minor-Ansatz entspricht dem Level III. Geschont werden bei der Axilladissektion der Nervus thoracicus longus, das thorakodorsale Nerven-/Gefäßbündel und möglichst auch der Nervus intercostobrachialis bzw. einzelne Äste dieses Nervs. Der Zugang zur Axilla erfolgt entweder im Rahmen eines ablativen Vorgehens über die Mastektomienarbe oder durch einen gesonderten axillären Schnitt.

Zu den häufigen Akutkomplikationen der Standard-Axilladissektion gehört das Serom sowie Taubheitsgefühl und Parästhesien im Bereich der Oberarminnenseite im Ausbreitungsgebiet des Nervus intercostobrachialis, gelegentlich auch Schmerzen. Langfristig, und ebenfalls gar nicht so selten, ist das Lymphödem des Armes zu beobachten. Seltener sind Bewegungseinschränkungen in der Schulter, Thrombosen im Bereich der Vena axillaris sowie Schädigungen von motorischen Nerven. Insbesondere ist für die Patientin das chronische Lymphödem des Armes eine erhebliche Belastung, das man auch in neueren Untersuchungen mit objektivierbaren Untersuchungsparametern in 10-25,5\% der Fälle findet.

Der axilläre Lymphknotenstatus ist auch heute noch der wichtigste prognostische Faktor für das rezidivfreie und das Gesamtüberleben. Nicht nur der qualitative Unterschied zwischen nodal-positiven und nodal-negativen Patientinnen, sondern auch die Anzahl der befallenen Lymphknoten bei positiver Axilla trennen prognostische Gruppen. Trotzdem ist der Lymphknotenstatus in den letzten Jahren etwas in den Hintergrund getreten. Dies liegt zum einen daran, daß kein wirklicher qualitativer Unterschied zwischen nodal-negativen und nodal-positiven Patientinnen besteht. So zeigen bei einem indirekten Vergleich adjuvant systemisch behandelte Patientinnen mit 1-3 befallenen Lymphknoten und einem histologischen Malignitätsgrad I oder II eine vergleichbar gute Prognose wie nodal-negative Patientinnen mit einem histologischen Grad-III-Tumor. Folgt man den Empfehlungen der Konsensus-Konferenz von St. Gallen, 1998, bleiben zudem weniger als 5\% der Patientinnen mit primärem Mammakarzinom ohne adjuvante systemische Therapie.

Eine weniger radikale Axilladissektion - bis hin zum vollständigen Verzicht auf die Axilladissektion - ist aber nur dann vertretbar, wenn das nicht mit einer Verschlechterung der Prognose einhergeht, die Axilladissektion selbst also in dem

\begin{tabular}{ll}
\hline KARGER & (1) 1999 S. Karger GmbH, Freiburg \\
Fax +49 7614520714 & Accessible online at: \\
$\begin{array}{l}\text { E-mail kargergmbh@aol.com } \\
\text { www.karger.com }\end{array}$ & http://BioMedNet.com/karger
\end{tabular}

Holger Eidtmann

Universitäts-Frauenklinik

Michaelisstraße 16

D-24105 Kiel 
entsprechenden Fall keine therapeutische Bedeutung hätte. Die Vorstellung, daß - abgesehen von den axillären Rezidiven - die Lymphonodektomie, wenn überhaupt, nur einen sehr beschränkten Einfluß auf die Prognose hat (d.h. die Abkehr von der alten Halstedtschen Hypothese hin zur Fisher-Hypothese des Brustkrebses als primär systemischer Erkrankung), basiert vor allem auf einer frühen Studie der NSABP. In dieser NSABP B04-Studie wurden randomisiert prospektiv Patientinnen mit klinisch nodal-negativen Mammakarzinomen untersucht. Verglichen wurde bei 1.079 Patientinnen eine radikale Mastektomie mit axillärer Lymphonodektomie, eine einfache Mastektomie mit Bestrahlung von Thoraxwand und Lymphabflußwegen und die alleinige totale Mastektomie. Trotz klinisch negativer Axilla fand sich in der Gruppe mit Lymphonodektomie in $40 \%$ der Fälle eine histologisch positive Axilla. Aufgrund der Randomisation muß auch ein ähnlicher Anteil in den anderen Gruppen angenommen werden. Hier wurde nur bei Verdacht auf ein axilläres Rezidiv im Rahmen der Nachbeobachtung eine axilläre Lymphonodektomie durchgeführt. Nach 10jährigem Follow-up fand sich kein signifikanter Unterschied hinsichtlich krankheitsfreiem, fernmetastasenfreiem und Gesamtüberleben [1]. Die Autoren zogen hieraus den Schluß, daß die operative Radikalität, insbesondere im Bereich der Axilla, den Krankheitsverlauf nur bedingt beeinflussen kann und daß die axillären Lymphknotenmetastasen weniger im Sinne einer Filterfunktion zu sehen sind, sondern als Indikatoren einer systemischen Verbreitung der Erkrankung.

Wir haben die Frage, ob die Radikalität der Axilladissektion prognostische Bedeutung beim nodal-negativen Mammakarzinom hat, retrospektiv bei nodal-negativen Patientinnen untersucht. Insgesamt wurden 1.140 Patientinnen in die Untersuchung aufgenommen. Patientinnen mit weniger als 6 Monaten Follow-up, brusterhaltend behandelte Patientinnen ohne ansonsten obligat durchgeführte Bestrahlung der Brust und einzelne Patientinnen, bei denen die Zahl der entfernten Lymphknoten nicht bekannt war, waren aus der weiteren Untersuchung ausgeschlossen worden, so daß in die Analyse insgesamt 1.029 Patientinnen eingingen. Bei diesen Patientinnen traten in einer medianen Nachbeobachtungszeit von 4 Jahren in 132 Fällen distante Metastasen auf, in 86 Fällen Lokalrezidive. Insgesamt waren von einer distanten Metastasierung oder einem Lokalrezidiv 180 Patientinnen betroffen, 67 dieser Patientinnen verstarben. Bei allen Patientinnen folgte die Aufarbeitung des Axillapräparates einem einheitlichen Vorgehen innerhalb einer Institution. Alle Patientinnen waren danach histologisch nodal-negativ einklassifiziert.

Die Gesamtgruppe der 1.029 Patientinnen wurde entsprechend einem Grenzwert der entnommenen Lymphknoten geteilt und die Gruppen hinsichtlich des 5 Jahre rezidivfreien Überlebens untersucht. Überraschenderweise fanden wir, egal wo wir den Grenzwert legten, also beispielsweise bei 8, 9,10,11 oder auch 16, 17, 18, 19 entnommenen Lymphknoten, daß immer die Gruppe mit mehr entnommenen Lymphknoten prognostisch besser war als die Gruppe mit weniger entfernten Lymphknoten. Es gab keinen Grenzwert, nach dem man sagen konnte, die Entfernung von mehr Lymphknoten hat keinen weiteren prognostischen Einfluß. Das galt auch, wenn wir entsprechend dem geltenden Standard nur diejenigen Patien- tinnen berücksichtigten, bei denen mindestens 10 Lymphknoten entfernt worden waren. So konnten wir beispielsweise nachweisen, daß die Patientinnen mit 17 und mehr entfernten Lymphknoten ein signifikant besseres rezidivfreies Überleben hatten als diejenigen mit 10 bis 16 entfernten Lymphknoten.

Diese Ergebnisse lassen sich auf mindestens 3 verschiedene Weisen interpretieren:

1. Je mehr Lymphknoten entfernt und untersucht werden, desto repräsentativer ist die Aussage nodal-negativ, falls keine Lymphknoten betroffen sind; oder

2. je mehr Lymphknoten entfernt worden sind, desto sicherer sind auch die «richtigen», also möglicherweise die SentinelLymphknoten, entfernt worden und damit auch die Aussage nodal-negativ korrekt; oder

3. die axilläre Lymphonodektomie auch bei tatsächlich nodalnegativer Axilla hat möglicherweise durch eine Veränderung der Abflußverhältnisse einen therapeutischen Einfluß auf die Erkrankung selbst.

\section{Die Sentinel Lymphnode Biopsy (SLN-Biopsie)}

Unter dem Sentinel-Lymphknoten oder auch Pförtnerlymphknoten versteht man den ersten das Tumorbett drainierenden Lymphknoten. Vorstellung ist, daß, wenn eine lymphogene Metastasierung stattfindet, diese kontinuierlich erfolgt ohne Überspringen einzelner Lymphknotenstationen, also ohne sogenannte Skip-Metastasierung. Unter dieser Voraussetzung wäre eine Prädiktion des Nodalstatus allein aufgrund der Untersuchung dieses Sentinel-Lymphknotens möglich.

Es sind inzwischen verschiedene Methoden zur Detektion des SLN beschrieben. Eine Möglichkeit ist die intratumorale, peritumorale oder oberhalb des Tumors subepidermale Injektion eines über die Lymphwege abfließenden blauen Farbstoffs (Patent-Blau oder Lymphazurin). Eine weitere Möglichkeit ist eine gleichartige Injektion eines radioaktiven markierten, kolloidalen Humanalbumins (Nanucoll, Albures). Während im ersteren Fall die Detektion des Sentinel-Lymphknotens erst nach Eröffnung der Axilla und Aufsuchen eines blaumarkierten Lymphknotens gelingt, ist bei der nuklearmedizinischen Methode die Detektion szintigraphisch oder präoperativ/intraoperativ mittels einer Sonde transkutan möglich.

Die Blaumarkierung hat den Vorteil, daß es zu keiner radioaktiven Belastung von Patientinnen und Personal kommt, sie an jeder Klinik durchführbar ist und je nach verwendetem Blaufarbstoff einen geringeren Zeit- und Kostenaufwand erfordert. Nachteile gegenüber der Technetium-Methode sind der größere operative Zugang, der notwendig wird, um einen Lymphknoten zu entdecken, die schwierige, weil subjektive Beurteilung des SLN, die teilweise wochenlang anhaltende Tätowierung aufgrund der Blauinjektion mit auch Blauverfärbung von Urin und Serum und die Tatsache, daß keine Aussage über einen parasternalen Abfluß möglich ist.

Die Vorteile der Technetium-Methode liegen in der schnellen transkutanen Lokalisation des SLN, dem geringen operativen Trauma, da nur eine kleine Biopsie notwendig ist, der Möglichkeit des gezielten Aufsuchens des Lymphknotens intraoperativ mittels Sonde und der Möglichkeit, auch über einen parasternalen Abfluß Information zu gewinnen. Nachteilig ist, 
Abb. 1. Design der geplanten deutschen Multizenter-Studie zur Wertigkeit der SLN-Biopsie (Randomisationsphase).

\begin{tabular}{lrr}
\hline & $\mathrm{n}$ & $\%$ \\
\hline Gesamtzahl & 124 & 100 \\
Darstellung des SLN & 107 & 87 \\
Tumorbefall & 45 & 36 \\
Nur SLN & 16 & 13 \\
Skip-Metastasen & 2 & 2 \\
Richtige Vorhersage & 98 & \\
Falsch-negativ & 4 & \\
\hline
\end{tabular}

daß die Technetium-Methode natürlich nur an Kliniken mit einer Nuklearmedizin durchführbar ist, sie einen höheren logistischen Aufwand auch im Hinblick auf die interdisziplinäre Kooperation notwendig macht und die allerdings geringe radioaktive Belastung von Patientinnen und Personal.

In unserer Klinik wurden bisher über 130 SLN-Biopsien in Kooperation mit der Universitätsklinik für Nuklearmedizin Kiel (Direktor Prof. Dr. Henze) durchgeführt, ohne daß wir bisher therapeutische Konsequenzen daraus gezogen hätten. Bei allen diesen Patientinnen wurde in gleicher Sitzung die Operation fortgeführt im Sinne einer Standard-Axilladissektion. Über die Ergebnisse unserer Klinik informiert Tabelle 1. Mit einer 98\%igen richtigen und einer 4\%igen falsch-negativen Vorhersage bewegen sich unsere Ergebnisse im Rahmen des internationalen Vergleichs [2].

Die SLN-Biopsie ist damit tatsächlich in der Lage, mit hinreichend hoher Wahrscheinlichkeit den axillären Lymphknotenstatus vorherzusagen.

Nach unseren Erfahrungen sind einige Punkte dabei allerdings $\mathrm{zu}$ beachten. So gelingt der intraoperative Nachweis eines Lymphabflusses häufig dann nicht, wenn Lymphknoten in der Axilla metastatisch befallen sind oder eine ausgeprägte Lymphangiosis carcinomatosa vorhanden ist, so daß faktisch die Lymphabflußwege obliterieren. Gleiches gilt für ausgedehnte Voroperationen im Bereich der Brust mit entsprechenden
Narbenbildungen. Im schlimmsten Fall kann bei metastatischer Durchsetzung eines Sentinel-Lymphknotens die Darstellung dieses Lymphknotens mißlingen bei gleichzeitigem Abfluß über einen anderen Lymphweg, so daß eventuell dieser als SNL erkannte Lymphknoten falsch-negativ ist. Um dieses Risiko zu minimieren, ist es nach unserer Erfahrung notwendig, nur klinisch nodal-negative Karzinome einzubeziehen, die auch von den sonstigen Tumorparametern mit hoher Wahrscheinlichkeit eine negative Axilla vorhersehen lassen. Dies betrifft vor allem große Tumore und eine histologisch nachgewiesene Lymphangiosis carcinomatosa peritumoral. Daneben muß, auch wenn der entsprechende Lymphknoten nicht markiert ist, ein intraoperativ auffälliger Lymphknoten bei der SLN-Biopsie entfernt werden.

\section{Geplante Studie}

Gemeinsam mit der Universitäts-Frauenklinik München, Großhadern, und der Universitäts-Frauenklinik Ulm planen wir eine multizentrische, prospektiv randomisierte Studie zur Frage, ob bei Patientinnen mit primärem Mammakarzinom und negativer SLN-Biopsie auf eine konventionelle StandardAxilladissektion verzichtet werden kann. Ziele dieser Studie sind neben der Überprüfung der Detektionsrate des SentinelLymphknotens und des prädiktiven Wertes seiner Histologie für den axillären Nodalstatus, der Vergleich der Morbidität nach einer alleinigen SLN-Biopsie gegenüber einer StandardAxilladissektion sowie der Äquivalenznachweis hinsichtlich rezidivfreiem und Gesamtüberleben zwischen diesen beiden Operationsmethoden. Die Studie selbst wird zweiphasig aufgebaut sein. Erst nach erfolgreichem Abschluß einer Trainingsphase pro teilnehmendem Zentrum wird dieses Zentrum in der Lage sein, an dem randomisierten Teil der Studie teilzunehmen. In der Trainingsphase soll bei mindestens 50 Patientinnen eine SLN-Biopsie durchgeführt werden, gefolgt von einer 
sofortigen Standard-Axilladissektion. Wird in dem Zentrum eine mindestens $80 \%$ ige Detektionsrate erreicht und zeigen die Ergebnisse bei höchstens 2 der letzten 30 operierten Patientinnen eine falsch-negative SLN-Biopsie, sind die Erfolgskriterien für den Abschluß der Trainingsphase erreicht. Über das Design der Randomisationsphase dieser Studie informiert Abbildung 1. Sind die Patientinnen SLN-positiv, folgt eine Standard-Axilladissektion. Sind sie SLN-negativ, folgt entsprechend der präoperativen Randomisation entweder eine Standard-Axilladissektion oder die weitere alleinige Kontrolle. Die Nachbeobachtung ist auf 5 Jahre angelegt. Gelingt keine Detektion des SLN, muß obligat eine Standard-Axilladissektion durchgeführt werden.

Unter der Voraussetzung, daß viele Kliniken an dieser Studie teilnehmen werden, besteht die Chance, innerhalb einer über- schaubaren Zeit zu überprüfen, ob eine alleinige SLN-Biopsie bei negativem SLN künftig ausreichen wird und es uns damit gelingt, nach Einführung der brusterhaltenden Therapie in den 70er Jahren nun die operative Radikalität im Sinne unserer Patientinnen weiter minimieren zu können.

\section{Literatur}

1 Fisher B, Redmond C, Fisher ER, Bauer M, Wolmark N, Wickerham DL, Deutsch M, Montague E, Margolese R, Foster R: Ten-year results of a randomized clinical trial comparing radical mastectomy and total mastectomy with or without radiation. N Eng J Med 1985;312:674-681.

2 McMasters KM, Giuliano AE, Ross MI, Reintgen DS, Hunt KK, Byrd DR, Klimberg VS, Whitworth PW, Tafra LC, Edwards MJ: Sentinel-lymph-node biopsy for breast cancer - not yet the standard of care. N Eng J Med 1998;339:990-995. 